\title{
Improvement of cyclodextrin glycosyltransferase gene expression in Escherichia coli by insertion of regulatory sequences involved in the promotion of RNA transcription
}

\begin{abstract}
Regulation of RNA transcription in controlling the expression of genes at promoter and terminator regions is crucial as the interaction of RNA polymerase occurred at both sites. Gene encoding cyclodextrin glycosyltransferase (CGTase) from Bacillus sp. NR5 UPM isolated in the previous study was used for further construction of pTZCGT-SS, pTZCGT-BS and pTZCGT-BT expression systems for enhancement of CGTase production. The putative promoter regions, $\bar{\imath} 35$ and $\bar{\imath} 10$ sequences were found in the upstream of the mature gene start codon. Whereas, long inverted repeats sequences which can form a stable stem and loop structure was found downstream of the open reading frame (ORF) of Bacillus sp. NR5 UPM CGTase. The construction of E. coli strain harbouring pTZCGT-BS showed increment of 3.2fold in CGTase activity compared to the wild type producer. However, insertion of terminator downstream of CGTase gene in E. coli strain harbouring pTZCGT-BT only resulted in 4.42 $\%$ increment of CGTase production compared to E. coli strain containing pTZCGT-BS, perhaps due to low intrinsic termination efficiency. Thus, it is suggested that the insertion of the putative promoter regions upstream of the coding sequence for the construction of CGTase expression system will further enhance in the recombinant enzyme production.
\end{abstract}

Keyword: Bacillus sp. NR5 UPM; CGTase expression system; Cyclodextrin glycosyltransferase (CGTase); Escherichia coli; Putative promoter regions 